\title{
Catabolic efficiency of aerobic glycolysis: The Warburg effect revisited
}

\author{
Alexei Vazquez ${ }^{* 1}$, Jiangxia Liu², Yi Zhou² and Zoltán N Oltvai
}

\begin{abstract}
Background: Cancer cells simultaneously exhibit glycolysis with lactate secretion and mitochondrial respiration even in the presence of oxygen, a phenomenon known as the Warburg effect. The maintenance of this mixed metabolic phenotype is seemingly counterintuitive given that aerobic glycolysis is far less efficient in terms of ATP yield per moles of glucose than mitochondrial respiration.

Results: Here, we resolve this apparent contradiction by expanding the notion of metabolic efficiency. We study a reduced flux balance model of ATP production that is constrained by the glucose uptake capacity and by the solvent capacity of the cell's cytoplasm, the latter quantifying the maximum amount of macromolecules that can occupy the intracellular space. At low glucose uptake rates we find that mitochondrial respiration is indeed the most efficient pathway for ATP generation. Above a threshold glucose uptake rate, however, a gradual activation of aerobic glycolysis and slight decrease of mitochondrial respiration results in the highest rate of ATP production.

Conclusions: Our analyses indicate that the Warburg effect is a favorable catabolic state for all rapidly proliferating mammalian cells with high glucose uptake capacity. It arises because while aerobic glycolysis is less efficient than mitochondrial respiration in terms of ATP yield per glucose uptake, it is more efficient in terms of the required solvent capacity. These results may have direct relevance to chemotherapeutic strategies attempting to target cancer metabolism.
\end{abstract}

\section{Background}

Since its original discovery by Warburg [1] it has been well established that most, if not all cancer cells are more dependent on aerobic glycolysis for ATP production than normal cells. The near uniform presence of this metabolic phenotype in tumor cells is counterintuitive, as glycolysis produces only 2 moles of ATP per mole of glucose, far less than the 36 generated by mitochondrial respiration (Figure 1). Several hypotheses have been proposed for the maintenance of this seemingly wasteful catabolic state. At the cell population level, mitochondrial respiration malfunction and enhancement of glycolysis are thought to be a metabolic advantage under the intermittent hypoxia conditions experienced by pre-malignant and malignant tumor cells $[2,3]$. However, aerobic glycolysis is not found exclusively in cancer cells, but is also observed in rapidly dividing normal cells even under con-

* Correspondence: vazqueal@umdnj.edu

1 Department of Radiation Oncology, The Cancer Institute of New Jersey and UMDNJ-Robert Wood Johnson Medical School, New Brunswick, NJ, 08963, USA Full list of author information is available at the end of the article ditions of normoxia [4]. It is also widely believed that the glycolysis rate increases in order to match the increased anabolic needs of the rapidly proliferating cells for precursor metabolites [5]. Yet, a focus on the cell's anabolic needs alone neglects two important facts; First, in addition to precursor metabolites, growing cells need ATP to meet the energy demands of biosynthetic pathways. Second, in its original definition the Warburg effect refers to the increase in the glycolysis rate ending in the excretion of lactate, which does not contribute to the production of precursor metabolites.

The actual molecular mechanisms that lead to the enhanced aerobic glycolysis are increasingly well understood. Under physiological condition, for example, PI3K/ Akt signaling pathways play a critical role in promoting aerobic glycolysis in activated $\mathrm{T}$ cells in response to growth factors or cytokines stimulation [6]. In the pathophysiological condition of tumorigenesis, malfunction of mitochondrial respiration due to mitochondrial DNA mutations/deletions is an important contributing factor [7-10]. Also, p53, one of the most frequently 


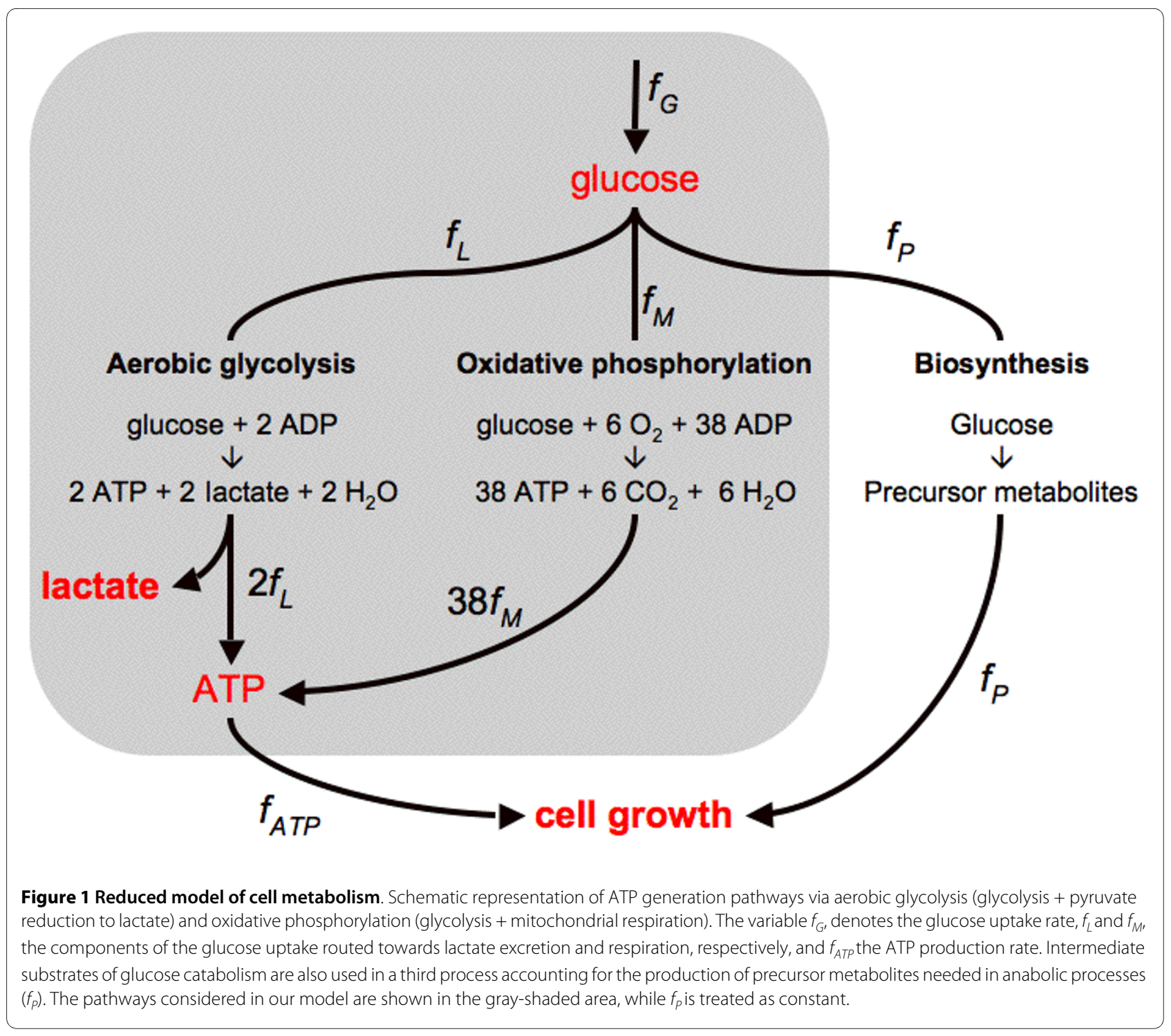

mutated genes in cancers, is both a positive regulator of mitochondrial respiration [11] and a negative regulator of glycolysis [12]. These, together with the activation of hypoxia-inducible factor (HIF), a transcription factor that is activated by hypoxic stress but also by oncogenic, metabolic, and oxidative stress, often lead to the overexpression of the glucose transporters and various glycolysis pathway enzymes or isozyme subtypes explaining the increased glucose uptake and altered utilization $[13,14]$.

The evidence accumulated so far thus indicates that the presence of aerobic glycolysis is a common characteristics of rapidly proliferating cells, and that it may offer a growth advantage to rapidly proliferating normal cells, e.g., during development and tissue regeneration, and to cancer cells in tumor formation. Yet, a system-level interpretation of the origin of this growth advantage has not yet been formally provided. To start addressing this issue, here we introduce a reduced flux balance model of ATP generation that incorporates a glucose uptake capacity constraint and the limited solvent capacity of the cell cytoplasm. The model allows us to uncover the existence of two different energetically favorable metabolic regimes within proliferating cells, a finding that is congruent with experimental results.

\section{Results}

\section{Reduced flux balance model of cell metabolism}

Figure 1 depicts a schematic model of glycolysis and mitochondrial respiration, the main pathways for ATP generation in cells. The glucose uptake flux $\left(f_{G}\right)$ is partitioned into the flux of aerobic glycolysis $\left(f_{L}\right)$, and to oxidative phosphorylation $\left(f_{M}\right)$. Aerobic glycolysis represents 
glycolysis, converting glucose into pyruvate, and then pyruvate reduction by lactate dehydrogenase (LDH) in the cytosol, resulting in the end product lactate that is then excreted to the extracellular millieau. ATP generation through oxidative phosphorylation is decomposed into the generation of pyruvate through glycolysis, followed by the oxidation of pyruvate in the TCA cycle and the respiratory chain, the latter two processes taking place in the cell's mitochondria. There is also a third component for glucose utilization accounting for the production of precursor metabolites needed in anabolic processes $\left(f_{P}\right)$ (e.g., intermediate metabolites of the pentose phosphate pathway and the TCA cycle [5]), which have to have an overall proportionality with the available energy currencies (phosphate donors, mainly ATP) to enable cell growth. Thus, in the absence of a full scale kinetic model, in our modeling we assume that the ATP production rate $\left(f_{A T P}\right)$ is proportional with the rate of anabolic processes $\left(f_{P}\right)$, and use $f_{A T P}$ as a surrogate for the overall cell metabolic rate (Figure 1).

Aerobic glycolysis includes the glycolysis pathway producing pyruvate and the pyruvate reduction producing lactate. The overall reaction for aerobic glycolysis is

$$
\mathrm{GLC}+2 \mathrm{ADP} \rightarrow 2 \mathrm{ATP}+2 \mathrm{LAC}+2 \mathrm{H}_{2} \mathrm{O}
$$

Aerobic glycolysis has a yield of 2 moles of ATP per mole of glucose, resulting from the conversion of glucose into lactate. On the other hand, oxidative phosphorylation includes the glycolysis pathway producing pyruvate, the oxidation of pyruvate in the TCA cycle, and the respiratory chain. The overall reaction of for oxidative phosphorylation is

$$
\mathrm{GLC}+6 \mathrm{O}_{2}+38 \mathrm{ADP} \rightarrow 38 \mathrm{ATP}+6 \mathrm{CO}_{2}+6 \mathrm{H}_{2} \mathrm{O}
$$

Oxidative phosphorylation yields 38 moles of ATP per mole of glucose, two of which are produced in glycolysis and the other 36 during the oxidation of pyruvate in the TCA cycle coupled to respiratory chain activity. Summing up these differential contributions we obtain the rate of ATP production

$$
f_{\text {ATP }}=2 f_{G}+36 f_{M}=2 f_{L}+38 f_{M},
$$

where the second equality was obtained using $f_{G}=f_{L}+$ $f_{M}$.

Our aim is to determine the optimal flux distribution $\left(f_{L}, f_{M}\right)$ that provides maximum ATP production rate given the cell's metabolic constraints. Of these, the first metabolic constraint is associated with the maximum glucose uptake rate (or glucose uptake capacity)

$$
f_{L}+f_{M}=f_{G} \leq F_{G}
$$

where $F_{G}$ is the maximum glucose uptake rate. The second constraint, quantified below, reflects on the high concentration of macromolecules within the cell's cytoplasm $[15,16]$, resulting in a limited solvent capacity for the allocation of metabolic enzymes. Enzyme molecules have a finite volume and the total sum of their volumes cannot exceed the cell volume. In our case, this constraint applies to the volume occupied by glycolytic enzymes, LDH and mitochondria. Enzymes associated with other pathways occupy a fraction of the intracellular volume as well. Nevertheless, this fraction simply restricts the amount of the cytoplasmic space available to glycolytic enzymes, LDH and mitochondria. More precisely, if $V_{G}$, $V_{L}$ and $V_{M}$ are the cell volume occupied by glycolytic enzymes, LDH and mitochondria, respectively, then

$$
V_{G}+V_{L}+V_{M} \leq V_{\text {ATP }}
$$

where $V_{A T P}$ is the total cell volume reserved for the allocation of components of the ATP producing pathways. The occupied volumes $V_{G}, V_{L}$ and $V_{M}$ are proportional to the enzyme masses $M_{G}, M_{L}$ and $M_{M}$, with $V_{G}=v_{G} M_{G}, V_{L}$ $=v_{L} M_{L}$ and $V_{M}=v_{M} M_{M}$, where $v_{G}, v_{L}$ and $v_{M}$ are the specific volumes of glycolytic enzymes, LDH and mitochondria, respectively. In turn, the glycolytic rate $\left(f_{G}\right)$, the lactate excretion rate $\left(2 f_{L}\right)$ and the mitochondrial ATP production rate $\left(36 f_{M}\right)$ are proportional to the mass of glycolytic enzymes, lactate dehydrogenase and mitochondria respectively, with $f_{G}=r_{G} M_{G} / V, 2 f_{L}=r_{L} M_{L} / V$ and $36 f_{M}=r_{M} M_{M} / V$, where $r_{G}$ is the glycolytsis rate per unit of glycolytic enzyme mass, $r_{L}$ is the rate of lactate production per mass of LDH, $r_{M}$ is the mitochondrial ATP production rate per unit of mitochondrial mass, and $V$ is the cell volume. In these equations the product by the mass and the division by the cell volume takes into account that the rates $r$ are commonly reported in the literature per unit of dry weight, while the pathway rates $f$ are reported per unit of cell volume. Because of the interdependency of volume, mass and reaction rate, the volume constraint (5) can be translated to the metabolic constraint

$$
\left(a_{L}+a_{G}\right) f_{L}+\left(a_{M}+a_{G}\right) f_{M} \leq \phi_{\text {ATP }}
$$

where $\varphi_{A T P}=V_{A T P} / V$ is the total volume fraction of the cell cytoplasm occupied by glycolytic enzymes, LDH and mitochondria, and the crowding coefficients $a_{G}=v_{G} / r_{G}, a_{L}$ $=2 v_{L} / r_{L}$ and $a_{M}=36 v_{M} / r_{M}$ quantify the occupied volume fractions per unit of glycolytic, lactate excretion and mitochondrial respiration rate, respectively. Using empirical data reported in the literature we have estimated the 
crowding coefficients (see Methods). We obtain $a_{G} \approx$ $0.0027(\mathrm{~min} / \mathrm{mM}), a_{L \approx} 0.00023(\mathrm{~min} / \mathrm{mM})$ and $a_{M} \approx 0.10$ $(\mathrm{min} / \mathrm{mM})$, indicating that the mitochondria contributes about 5 and 50 times more to molecular crowding than glycolytic enzymes and lactate dehydrogenase, respectively. The unexpected consequences derived from this fact will be uncovered below.

In our modeling we assume that $V_{A T P}, r_{G}, r_{L}$ and $r_{M}$ are constant parameters that can be obtained from experimental estimates. Note though, that this is an approximation, as there may be regulatory mechanisms that under certain environmental or developmental conditions are capable of altering the amount of intracellular space allocated to ATP producing pathways and the activity of glycolytic enzymes, LDH and mitochondria.

Taken together the relevant metabolic optimization problem is as follows: maximize the ATP production rate (3) under the metabolic capacity constraints (4) and (6).

\section{Optimal solution}

In the following we discuss the model-predicted utilization of aerobic glycolysis and oxidative phosphorylation pathways in the context of the cell's glucose uptake rate. It becomes obvious that the glycolysis rate matches the maximum glucose uptake capacity $\left(f_{G}=F_{G}\right)$ to maximize the ATP production rate. However, there are some differences in the flux distribution along aerobic glycolysis and oxidative phosphorylation depending on the glucose uptake rate. Specifically,

$$
\begin{aligned}
& f_{M}=f_{G} \\
& f_{L}=0
\end{aligned}
$$

for $f_{G}<f_{1}$, and for $f_{G} \geq f_{1}$

$$
\begin{aligned}
& f_{M}=\left(a_{L}+a_{G}\right)\left(f_{2}-f_{G}\right) /\left(a_{M}-a_{L}\right) \\
& f_{L}=\left(a_{M}+a_{G}\right)\left(f_{G}-f_{1}\right) /\left(a_{M}-a_{L}\right)
\end{aligned}
$$

where

$$
\begin{aligned}
& f_{1}=\phi_{\text {ATP }} /\left(a_{M}+a_{G}\right) \\
& f_{2}=\phi_{\text {ATP }} /\left(a_{L}+a_{G}\right) .
\end{aligned}
$$

The optimal solution is graphically illustrated in Figure $2 \mathrm{a}$ and $2 \mathrm{~b}$ (lines). At low glucose uptake rates ATP is entirely produced by the oxidative phosphorylation pathway and there is no lactate production (and excretion). This trend continues up to a threshold glucose uptake rate, $f_{1}(9)$, when mitochondria occupy the entire cell volume fraction $\left(V_{A T P}\right)$ available for ATP production pathways (Figure 2a, blue dashed line, white background, glucose-limited regime). Beyond this threshold value the concentration of mitochondria, and therefore the rate of ATP production through oxidative phosphorylation, cannot be increased further. However, additional glucose uptake may be diverted toward pathway(s) less efficient in terms of ATP yield per mole of glucose. Indeed, the optimal solution predicts a metabolic switch, in which glucose uptake rates larger than $f_{1}$ leads to a linearly increasing lactate production (Figure 2a, red solid line, gray background, space-limited regime), and therefore a component of the ATP production is now derived from aerobic glycolysis. Furthermore, the increase in lactate production is accompanied by a slight, gradual reduction of the oxidative phosphorylation flux (Figure 2a, blue dashed line, gray background). Therefore, due to the increased aerobic glycolysis rate ATP production can still be increased beyond $f_{1}$ (Figure $2 \mathrm{~b}$, green dotted line). However, because aerobic glycolysis has a lower yield than oxidative phosphorylation, the ATP production rate increases with a lower slope beyond $f_{1}$. Finally, it is worth noting that because $a_{M}$ is 5 and 10 times higher than $a_{G}$ and $a_{L}$, respectively, these plots are not too sensitive to the precise estimates of the crowding coefficients. Indeed, assuming that $a_{M}$ is much greater than $a_{G}$ and $a_{L}$, we can approximate (8) by $f_{M \approx} f_{1}$ and $f_{L \approx} f_{G}-f_{1}$. Therefore, we predict that the plots of $f_{M}$ and $f_{L}$ versus $f_{G}$ are rather universal, with a distinctive change of behavior at $f_{G}=f_{1}$.

\section{Experimental evidence for model validity}

The model described above results in two experimentally testable predictions: the glucose uptake threshold $f_{1}(9)$, where the metabolic switch takes place, and the shape of the lactate excretion, oxidative phosphorylation and ATP production plots as a function of the glucose uptake rate. To start addressing the validity of these predictions we first utilized previously reported experimental data for mouse LS cells [17] (Figure 2a, b), hybridoma cells [18] (Figure 2c), and a mixture of cancer and normal cells [4] (Figure 2d), indicating that cancer cells and normal cells follow the same law.

In the first two cases $[17,18]$, the growth rate of the cells were manipulated by their growth in chemostat cultures at different dilution rates, a protocol that is known to substantially vary the cells' glucose uptake rate. In both cases the empirical lactate excretion plots (Figure 2a, c, red circles) follow the model predictions (Figure 2a, c, red solid lines): at low glucose uptake rates there is no significant lactate excretion, but once reaching a threshold the lactate excretion rate increases linearly with the further increase in glucose uptake rate. Moreover, the slope of this linear increase coincides with that predicted by the model. For the LS cells, we can also derive their oxidative phosphorylation (Figure 2a, blue squares) and ATP production rate (Figure $2 \mathrm{~b}$, diamonds) from the published 

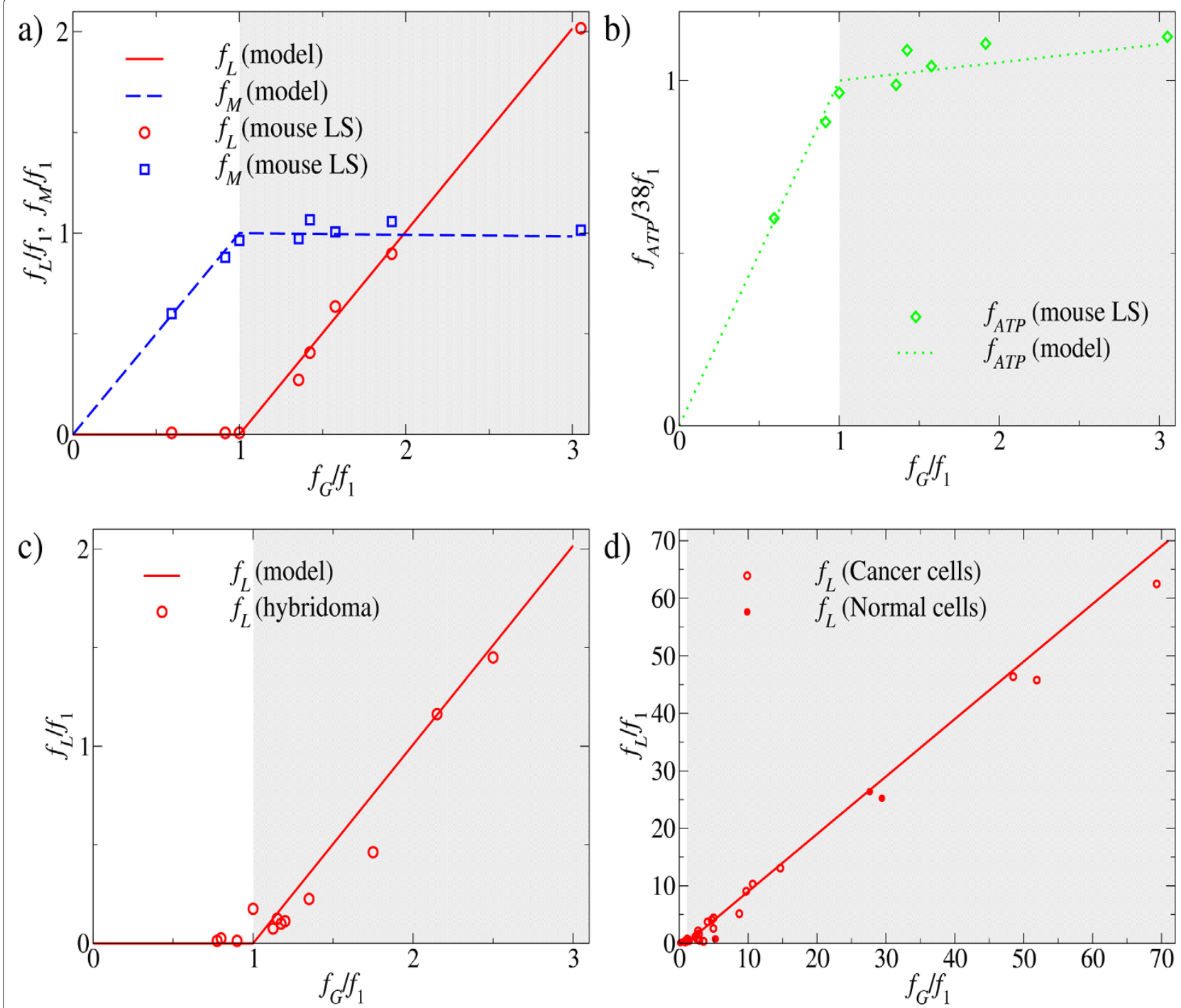

e)

\begin{tabular}{|c|c|c|c|c|}
\hline cell type & mouse $\mathrm{LS}$ & hybridoma & mixture & model \\
\hline $\mathrm{f}_{1}(\mathrm{mM} / \mathrm{min})$ & $0.8-1.0$ & $0.37-0.54$ & 1.4 & $0.7-3.8$ \\
\hline
\end{tabular}

Figure 2 Catabolic regimes within proliferating mammalian cells. a, b, c, d) Model-predicted (lines) and measured (symbols) fluxes as a function of the glucose uptake rate: a) and b) LS mouse cells, c) hybridoma cells and d) mixture of cancer and normal cells. The two different metabolic regimes are indicated by the white and gray backgrounds, respectively. All fluxes are reported in units of $f_{1}$, the glucose uptake rate threshold, reported in panel e).

experimental data [17] (see Additional file 1 Table S2 for details), and in both cases we obtain a good agreement between the experimental data (symbols) and the model predictions (lines). In the third case, the mixture of cancer and normal cells is a compilation of experimental reports using different methods and cell lines [4] (see also Additional file 1 Table S4). Here again, glucose uptake rates and lactate excretion levels show a good correlation to each other (Figure $2 \mathrm{~d}$ ).

The glucose uptake threshold, i.e., the uptake rate where the switch takes place, can be estimated from a linear fit to the lactate excretion plot in the regime where lactate is excreted (Additional file 2 Figure S1). It ranges between $0.8-1.0 \mathrm{mM} / \mathrm{min}$ for mouse LS cells, $0.37-0.54$ 
$\mathrm{mM} / \mathrm{min}$ for hybridoma cells and $1.4 \mathrm{mM} / \mathrm{min}$ for the mixture of cancer and normal cells (Figure 2e). Based on independent empirical estimates of the cell volume fraction occupied by mitochondria and the ATP production rate per unit of mitochondrial mass, our model predicts the threshold glucose uptake rate, $f_{1}$, to be in the range between 0.7 and $3.8 \mathrm{mM} / \mathrm{min}$ (Methods), which overlaps with the ranges reported above for the different cell lines. This agreement is surprisingly good given that they were estimated using independent data.

To further test the model's predictive capability, we have next examined human BJ fibroblast cells serially transduced with the catalytic domain of human telomerase (hTERT), SV40 large T (LT) and small T (ST) antigens, and an oncogenic allele of $\mathrm{H}$-ras $\left(\mathrm{H}-\mathrm{RasV}^{12}\right)$ [19]. Previous studies using these cells have shown, as we partially confirm here (Additional file 2 Figure S2), that the growth rate, soft agar colony formation, and in vivo tumorigenicity of CL1-[hTERT], CL2-[hTERT+LT], CL3[hTERT+LT+ST], and CL4-[hTERT+LT+ST+H-Ras] cells largely correspond with their level of transduction [19], and that their glucose uptake rates progressively increase from normal toward more tumorigenic transformants (CL1 to CL4)) [20]. Previous experiments also demonstrated that normal CL1 cells display a severe reduction in their ATP content upon inhibition of mitochondrial oxidative phosphorylation, while the fully tumorigenic CL4 cells remain largely unaffected (CL2 ad CL3 cells display an intermediate reduction in their ATP levels) [20]. In contrast, compared to the other three cell types CL4 cells display higher level of lactate production and a more severe reduction in their ATP content when their LDH activity is inhibited [20].

To confirm and extend the above findings, we experimentally tested the extracellular lactate levels, mitochondrial mass and mitochondrial membrane potential (a proxy for respiratory chain activity) of CL1-4 cells at $48 \mathrm{~h}$, $72 \mathrm{~h}, 96 \mathrm{~h}$, and $120 \mathrm{~h}$ after seeding the same amount of cells, along with cell counts and total protein concentration measurements. We find that the tumorigenic CL4 cells have the highest extracellular lactate levels compared to the other cell types at all time points tested that correlates with a concomitant change in LDH enzyme activity but not protein expression level (Additional file 2 Figure S3). CL4 cells also have the lowest mitochondrial mass and membrane potential, though compared to CL1 cells their decrease is larger than predicted by our model (Additional file 2 Figure S4) (a detailed description of the results is given in the Additional file 2). Taken together, the observed alterations in lactate production and mitochondrial parameters, together with the previously observed oxidative phosphorylation and LDH activity inhibition profiles in the same cells [20], are congruent with the model predictions: an increase of lactate excre- tion and decline of mitochondrial respiration with an increasing replicative/tumorigenic capacity (quantified by the ATP production rate in the model).

\section{Discussion and conclusions}

Rapidly proliferating mammalian cells, including cancer cells, almost always exhibit aerobic glycolysis even under normoxic conditions, characterized by an increased lactate excretion rate relative to the respiration rate. Since its original observation by Warburg [1], from the standpoint of metabolic efficiency this has been a surprising observation because aerobic glycolysis is far less efficient than mitochondrial respiration in terms of moles of ATP generated per mole of glucose. Implicit to this result, however, is the assumption that glucose uptake is a limiting factor of cell metabolism. Yet, the validity of this assumption on metabolic efficiency in the context of additional physicochemical constraints of the cell has not been formally addressed.

By developing a reduced flux balance model of ATP production (Figure 1) here we demonstrate the existence of two different metabolic regimes in mammalian cells, depending on whether the cell metabolic rate is limited by the glucose uptake or by the solvent capacity of the cell's cytoplasm. In conditions where glucose transport limits the cell's metabolic activity we find that the optimal solution is to produce ATP entirely via mitochondrial respiration, confirming that ATP generation yield is the appropriate measure within this regime; However, at high glucose uptake rates the optimal solution is characterized by an abrupt increase of the flux towards lactate production (and excretion) and a gradual decrease of mitochondrial respiration (Figure 2). This second regime is determined by the existence of a limited cytoplasmic solvent capacity for allocating the components of the ATP generating pathways (see equation (6)). For glucose uptake rates exceeding the threshold level, $f_{1}$, the cell cannot further increase the concentration of mitochondria to increase the respiration rate in order to match the increased glucose uptake rate. Yet, the glucose uptake "surplus" may now be diverted towards a pathway that is less efficient in terms of ATP yield per mole of glucose but is characterized by a higher rate of lactate (and thus ATP) production per its own (i.e., glycolytic and LDH enzymes) mass than the rate of ATP production by respiration per mitochondrial mass.

Comparisons to experimental data reported for LS mouse cells [17], hybridoma cells [18] and a collection of normal and cancer cells [4] provide an indirect support for the validity of these predictions. The agreement between the experimentally determined fluxes and the model predictions is remarkably good (see Figure 2). In all cases the empirical plot of the lactate excretion as a function of the glucose uptake rate (symbols) falls close to 
that predicted by the model (lines). More importantly, the experimental values indicate a glucose uptake threshold around $1 \mathrm{mM} / \mathrm{min}$, which falls in the range $f_{1} \sim 0.7-3.8$ $\mathrm{mM} / \mathrm{min}$ predicted by our model. Also, experimental data reported here and previously [20] on lactate production, mitochondrial parameters and enzyme inhibition profiles of genetically modified human BJ fibroblast cells [19] are congruent with the model's predictions. In similar recent experiments, oncogenic Ras/E1A transformed low passage murine embryonic fibroblast (MEFs) (RasLP) also displayed higher mitochondrial respiration activity and lower lactate secretion compared to more rapidly dividing high passage MEFs (Ras-HP) [21], and tumor progression in patients may correlate with reduced mitochondrial density and/or function [22-24].

The model described here has its limitations and does not account for all the potential population-level advantages of the Warburg effect. First, decreased mitochondrial respiration also contributes to the mitochondrial depolarization that is frequently observed in tumor cells, and that is thought to dampen the cell's propensity for mitochondrially-induced apoptosis [25]. Secondly, the effect of the secreted lactic acid on the surrounding normal tissue may increase the tumor cells' invasive potential [3]. Thus, tumor cells may evolve to decrease mitochondrial respiration (and increase glycolysis) even further than predicted by our model. Third, increased glycolytic rates can increase the sensitivity of the pathways producing precursor metabolites to specific regulators, allowing high rates of proliferation when required [26]. Moreover, our simplified model focuses on the ATP demand and the cell volume fraction occupied by mitochondria. However, in addition to ATP rapidly proliferating cells need increased amount of precursor metabolites to fuel their biosynthetic pathways [5,27]. Thus, ultimately a cell-scale metabolic model will need to be developed to take into account all the cell's metabolic demands. The achievement of this goal is currently limited by the unavailability of enzyme activity measurements at the human metabolic network scale.

From a more general perspective, it is evident that aerobic glycolysis is not unique to cancer- and rapidly proliferating normal mammalian cells but is present in unicellular organisms, as well. For instance, under aerobic growth conditions fast growing $E$. coli and S. cerevisiae cells also exhibit aerobic glycolysis, resulting in the excretion of the metabolic byproducts, acetate and ethanol, respectively. More importantly, both for $E$. coli and for $S$. cerevisiae a shift toward aerobic glycolysis follows a switch from limited to rich nutrient conditions [28,29], and for E. coli the maximum growth rates in different carbon sources and the mode of utilization of mixed carbon sources are in agreement with those allowed by the limited solvent capacity of the cell's cytoplasm $[29,30]$. Taken together with our current finding for mammalian cells, these results suggest that the appearance of aerobic glycolysis provides an energetically favorable catabolic state for all rapidly proliferating cells due to the inherent physicochemical constraint of molecular crowding on cell metabolism.

These results may have relevance to cancer therapy as well. It has been previously proposed that the Warburg effect may represent a unique property of cancer cell metabolism [13]. Pharmacological reduction of lactate production and excretion may indeed reduce the invasive potential of tumor cells or makes them more susceptible to apoptosis [25]. However, the metabolic state of tumor cells itself is not a unique target for cancer chemotherapy, though individual, tumor specific isozymes may be targeted [14].

\section{Methods}

\section{Parameters estimates: model}

$a_{G}$ : The glycolysis rate per total mass of glycolytic enzyme is about $r_{G}=0.29 \mathrm{mmol}$ glucose uptake $/ \mathrm{min} / \mathrm{g}$ at $30^{\circ} \mathrm{C}$ [31], obtained as $23 \mu \mathrm{mol} / \mathrm{min} / \mathrm{mL}$ lactate production rate (Table four [31]), divided by $40 \mathrm{mg} / \mathrm{mL}$ of total glycolytic protein concentration (Table two [31]), and divided by 2 to convert from lactate production to glucose uptake. The specific volume of glycolytic enzymes is estimated from the specific volume of globular proteins 0.79 $\mathrm{mL} / \mathrm{g}$ [32], thus we use $v_{G}=0.79 \mathrm{~mL} / \mathrm{g}$. Putting these two values together we obtain $a_{G}=v_{G} / r_{G}=0.0027(\mathrm{~min} / \mathrm{mM})$. $a_{L}$ : The rate of lactate production per mass of lactate dehydrogenase is about $r_{L}=7 \mathrm{mmol}$ lactate $/ \mathrm{min} / \mathrm{g}$ at $30^{\circ} \mathrm{C}$ [31], obtained as $23 \mu \mathrm{mol} / \mathrm{min} / \mathrm{mL}$ lactate production rate (Table four [31]), divided by $3.2 \mathrm{mg} / \mathrm{mL}$ of lactate dehydrogenase concentration (Table two [31]). The specific volume of lactate dehydrogenase is estimated from the specific volume of globular proteins $0.79 \mathrm{~mL} / \mathrm{g}$ [32], thus we use $v_{L}=0.79 \mathrm{~mL} / \mathrm{g}$. Putting these two values together we obtain $a_{L}=2 v_{L} / r_{L}=0.00023(\mathrm{~min} / \mathrm{mM}) . a_{M}$ : The mitochondrial rate of ATP generation per mitochondrial mass is in the range of 0.1-1.0 $\mathrm{mmol} \mathrm{ATP} / \mathrm{min} / \mathrm{g}$ [3335]. We use the maximum value $r_{M}=1.0 \mathrm{mmol} \mathrm{ATP} / \mathrm{min} /$ g. The mitochondrial specific volume has been reported to be $3.15 \mathrm{~mL} / \mathrm{g}$ in mammalian liver [36] and $2.6 \mathrm{~mL} / \mathrm{g}$ in muscle [37], respectively. We use the average between these two values $v_{M}=2.9 \mathrm{~mL} / \mathrm{g}$. Putting these two values together we obtain $a_{M}=36 v_{M} / r_{M}=0.10(\mathrm{~min} / \mathrm{mM}) . \varphi_{A T P}$ : The cell volume fraction occupied by cellular components involved in the generation of ATP was estimated from the cell volume fraction occupied by mitochondria. Literature reports for the latter range between 0.07 in some cell lines to 0.38 in muscle cells (Additional file 2 Table S1). Thus we use $\varphi_{\text {ATP }}=0.07-0.38 . f_{1}$ : Substituting 
the experimental estimates $a_{M}=0.17(\mathrm{~min} / \mathrm{mM}), a_{G}=$ $0.0027(\mathrm{~min} / \mathrm{mM})$ and $\varphi_{\text {ATP }}=0.07-0.38$ into (9) we obtain $f_{1}=0.7-3.8 \mathrm{mM} / \mathrm{min}$.

\section{Parameter estimates: flux data}

Murine LS cells: The experimental flux measurements for the mouse LS cell line were obtained from reference [17] (Additional file 1 Table S2), reporting the glucose uptake, lactate production and oxidized glucose rates, in units of $\mu$ mole per $10^{6}$ cells per day. The experimental value of $f_{1}$ was estimated from a linear fit to the data points in the region where there is a significant lactate production (Additional file 2 Figure S1a), resulting in $f_{1}=0.69 \mu$ mole per $10^{6}$ cell per day. Taking into account that LS mouse cells have a typical volume of $500-600 \mu \mathrm{m}^{3}$ [38] we obtain $f_{1}=1 \mathrm{mM} / \mathrm{min}$. Hybridoma cells: The experimental flux measurements for the hybridoma cell line were obtained from reference [18] (Additional file 2 Table S3), reporting the glucose uptake and lactate production rates, in units of mmole per $10^{9}$ cells per hour. The experimental value of $f_{1}$ was estimated from a linear fit to the data points in the region where there is a significant lactate production (Additional file 2 Figure S1b), resulting in $f_{1}=0.042$ mmole per $10^{6}$ cells per hour. Taking into account that hybridoma cells have a typical volume of $1300-1900 \mu \mathrm{m}^{3}$ [39] we obtain $f_{1}=0.37-0.54 \mathrm{mM} / \mathrm{min}$. Mixture of cancer and normal cells: The experimental flux measurements for the mixture of cancer and normal cells were obtained from reference [4] (Additional file 1 Table S4), reporting the glucose uptake and lactate production rates, in units of $\mathrm{mmol} / \mathrm{g} / \mathrm{h}$. The experimental value of $f_{1}$ was estimated from a linear fit to the data points in the region where there is a significant lactate production (Additional file 2 Figure S1c), resulting in $f_{1}=0.029 \mathrm{mmol} / \mathrm{h} / \mathrm{g}$. Assuming a typical cell density of $0.34 \mathrm{~g} / \mathrm{mL}$ we obtain $f_{1}=1.4 \mathrm{mM} /$ min.

\section{Experimental Protocols}

Genetically-manipulated derivatives of human BJ fibroblasts, transfected with the catalytic subunit of human telomerase (hTERT) (CL1), hTERT+ SV40 large T (LT) (CL2), hTERT+LT+ SV40 small T (ST)(CL3), and hTERT+LT+ST+ V12 mutant human Ras (Ras) (CL4) [19] were generous gifts from Dr. W. Hahn (Dana Farber Cancer Institute, Boston). The cells were maintained in DMEM supplemented with $25 \mathrm{mM}$ glucose, $4 \mathrm{mM}$ glutamine and $10 \% \mathrm{FBS}$ at $37^{\circ}$ in a $\mathrm{CO}_{2}$ incubator with $5 \%$ $\mathrm{CO}_{2}$ and $95 \%$ air. The experimental protocols for Western blots, Ras activity measurements cell growth and soft agar assays, LDH activity measurements, and for measuring mitochondrial mass and membrane potential are provided in the Additional file 2.

\section{Additional material}

\section{Additional file 1 Supplementary tables. Supplementary Tables S2, S3} and $\mathrm{S4}$

Additional file $\mathbf{2}$ Supplementary information. Experimental protocols and results (p2-4), Table S1 for parameter estimates (p6), Supplementary Figures (p6-8)

\section{Competing interests}

The authors declare that they have no competing interests.

\section{Authors' contributions}

AV and ZNO conceived the project. AV developed the metabolic model and analyzed the literature data. $J L$ and $Y Z$ performed the reported experiments. AV and ZNO wrote the manuscript. All authors read and approved the final manuscript.

\section{Acknowledgements}

AV was sponsored by the RWJ Foundation, Project title: "Strengthening the Cancer Institute of New Jersey in Cancer Prevention, Control and Population Science to Improve Cancer Care", Cancer Informatics Category. This work has been supported in part by NIH grants NIAID U01 Al070499 to ZNO.

\section{Author Details}

1Department of Radiation Oncology, The Cancer Institute of New Jersey and UMDNJ-Robert Wood Johnson Medical School, New Brunswick, NJ, 08963, USA and 2Department of Pathology, University of Pittsburgh, Pittsburgh, PA, 15261 USA

Received: 11 January 2010 Accepted: 6 May 2010

Published: 6 May 2010

\section{References}

1. Warburg O: On the origin of cancer cells. Science 1956, 123:309-314

2. Brahimi-Horn MC, Chiche J, Poyssegur J: Hypoxia signaling controls metabolic demand. Curr Opin Cell Biol 2007, 19:223-229.

3. Gatenby RA, Gillies RJ: Why do cancers have high aerobic glycolysis? Nature Rev Cancer 2004, 4:891-899.

4. Zu XL, Guppy M: Cancer metabolism: facts, fantasy, and fiction. Biochem Biophys Res Comm 2004, 313:459-465.

5. Heiden MG Vander, Cantley LC, Thompson CB: Understanding the Warburg Effect: The metabolic requirements of cell proliferation. Science 2009, 324:1029-1033.

6. Wieman HL, Wofford JA, Rathmell JC: Cytokine stimulation promotes glucose uptake via phosphatidylinositol-3 kinase/Akt regulation of Glut1 activity and trafficking. Mol Biol Cell 2007, 18:1437-1446.

7. Copeland WC, Wachsman JT, Johnson FM, Penta JS: Mitochondrial DNA alterations in cancer. Cancer Invest 2002, 20:557-569.

8. Nomoto S, Sanchez-Cespedes M, Sidransky D: Identification of mtDNA mutations in human cancer. Methods Mol Biol 2002, 197:107-117.

9. Carew JS, Zhou Y, Albitar M, Carew JD, Keating MJ, Huang P: Mitochondrial DNA mutations in primary leukemia cells after chemotherapy: clinical significance and therapeutic implications. Leukemia 2003, 17:1427-1447.

10. Pelicano H, Xu RH, Du M, Feng L, Sasaki R, Carew JS, Hu Y, Ramdas L, Hu L, Keating MJ, Zhang W, Plunkett W, Huang P: Mitochondrial respiration defects in cancer cells cause activation of Akt survival pathway through a redox-mediated mechanism. J Cell Biol 2006, 175:913-932.

11. Matoba S, Kang JG, Patino WD, Wragg A, Boehm M, Gavrilova O, Hurley PJ, Bunz F, Hwang PM: p53 regulates mitochondrial respiration. Science 2006, 312:1650-1653.

12. Bensaad K, Tsuruta A, Selak MA, Vidal MN, Bartrons R, Gottlieb E, Vousden $\mathrm{KH}$ : TIGAR, a p53-inducible regulator of glycolysis and apoptosis. Cell 2006, 126:117-120.

13. Kroemer G, Pouyssegur J: Tumor cell metabolism: cancer's Achilles' heel. Cancer Cell 2008, 13:472-482

14. Christofk HR, Heiden MG Vander, Harris MH, Ramanathan A, Gerszten RE, Wei R, Fleming MD, Schreiber SL, Cantley LC: The M2 splice isoform of pyruvate kinase is important for cancer metabolism and tumour growth. Nature 2008, 452:230-3. 
15. Ellis RJ: Macromolecular crowding: obvious but underappreciated. Trends Biochem Sci 2001, 26:597-604.

16. Hall D, Minton AP: Macromolecular crowding: qualitative and semiquantitative successes, quantitative challenges. Biochim Biophys Acta 2003, 1649:127-39.

17. Sinclair R: Response of mammalian cells to controlled growth rates in steady-state continuous culture. In Vitro 1974, 10:295-305.

18. Gambhir A, Korke R, Lee J, Fu P-C, Europa A, Hu W-S: Analysis of cellular metabolism of hybridoma cells at distinct physiological states. J Biosci Bioeng 2003, 95:317-327.

19. Hahn WC, Counter CM, Lundberg AS, Beijersbergen RL, Brooks MW, Weinberg RA: Creation of human tumour cells with defined genetic elements. Nature 1999, 400:464-468.

20. Ramanathan A, Wang C, Schreiber SL: Perturbational profiling of a cellline model of tumorigenesis by using metabolic measurements. Proc Natl Acad Sci USA 2005, 102:5992-7.

21. de Groof AJ, te Lindert MM, van Dommelen MM, Wu M, Willemse M, Smift AL, Winer M, Oerlemans F, Pluk H, Fransen JA, Wieringa B: Increased OXPHOS activity precedes rise in glycolytic rate in H-RasV12/E1A transformed fibroblasts that develop a Warburg phenotype. $\mathrm{Mol}$ Cancer 2009, 8:54

22. Sweeney MJ, Ashmore J, Morris HP, Weber G: Comparative biochemistry of hepatomas. IV. Isotope studies of glucose and fructose metabolism in liver tumors of different growth rates. Cancer Res 1963, 23:995-1002.

23. Lee HC, Yin PH, Lin JC, Wu CC, Chen CY, Wu CW, Chi CW, Tam TN, Wei YH: Mitochondrial genome instability and mtDNA depletion in human cancers. Ann N Y Acad Sci 2005, 1042:109-22.

24. Lin CS, Wang LS, Tsai CM, Wei YH: Low copy number and low oxidative damage of mitochondrial DNA are associated with tumor progression in lung cancer tissues after neoadjuvant chemotherapy. Interact Cardiovasc Thorac Surg 2008, 7:954-958

25. Michelakis ED, Webster L, Mackey JR: Dichloroacetate (DCA) as a potential metabolic-targeting therapy for cancer. Br J Cancer 2008, 99:989-994.

26. Newsholme EA, Crabtee B, Ardawi MSM: The role of high glycolysis and glutamine utilization in rapidly dividing cells. Biosci Rep 1985, 5:393-400.

27. DeBerardinis RJ, Lum JJ, Hatzivassiliou G, Thompson CB: The biology of cancer: metabolic reprogramming fuels cell growth and proliferation. Cell Metab 2008, 7:11-20.

28. Frick O, Wittmann C: Characterization of metabolic shift between oxidative and fermentative growth in Saccharomyces cerevisiae by comparative ${ }^{13} \mathrm{C}$ flux analysis. Microbial Cell Factories 2005, 4:30.

29. Vazquez A, Beg QK, de Menezes MA, Ernst J, Bar-Joseph Z, Barabási A-L, Boros LG, Oltvai ZN: Impact of the solvent capacity constraint on E. coli metabolism. BMC Systems Biol 2008, 2:7.

30. Beg QK, Vazquez A, Ernst J, de Menezes MA, Bar-Joseph Z, Barabási A-L, Oltvai ZN: Intracellular crowding of macromolecules defines the mode and sequence of substrate uptake by Escherichia coli and constrains its metabolic activity. Proc Natl Acad Sci USA 2007, 104:12663-8.

31. Scopes RK: Studies of reconstituted muscle glycolytic system. Biochem J 1973, 134:197-208.

32. Lee B: Calculation of volume fluctuations for globular protein models. Proc Natl Acad Sci USA 1983, 80:622-626.

33. Wibom R, Hultman E, Johansson M, Matherei K, Constantin-Teodosiu D, Schantz PG: Adaptation of mitochondrial ATP production in human skeletal muscle to endurance training and detraining. J Appl Physiol 1992, 73:2004-10

34. Short KR, Nygren J, Barazzoni R, Levine J, Nair KS: T3 increases mitochondrial production in oxidative muscle despite increased expression of UCP2 and -3. Am J Physiol Endocrinol Metab 2001 280:E761-E767.

35. Hou X-Y, Green S, Askew CD, Barker G, Green A, Walker PL: Skeletal muscle mitochondrial ATP production rate and walking performance in peripherical arterial disease. Clin Physiol Func Im 2002, 22:226-232.

36. Glass U, Bahr FG: Quantitative study of mitochondria in rat liver. $J$ Cell Biol 1966, 29:507-523.

37. Schwerzmann $\mathrm{K}$, Hoppeler $\mathrm{H}$, Kayar SR, Wibel ER: Oxidative capacity of muscle mitochondria. Proc Natl Acad Sci USA 1989, 86:1583-1587.

38. Blaker GJ, Pirt SJ: The uptake of vitamins by mouse fibroblast cells (strain LS) during growth in a chemically defined medium. J Cell Sci 1971, 8:709-725
39. Sen S, Srienc F, Hu W-S: Distinct volume distribution of viable and nonviable hybridoma cells: A flow cytometric study. Cytotechnology 1989, 2:85-94.

doi: $10.1186 / 1752-0509-4-58$

Cite this article as: Vazquez et al., Catabolic efficiency of aerobic glycolysis: The Warburg effect revisited BMC Systems Biology 2010, 4:58

\section{Submit your next manuscript to BioMed Central and take full advantage of:}

- Convenient online submission

- Thorough peer review

- No space constraints or color figure charges

- Immediate publication on acceptance

- Inclusion in PubMed, CAS, Scopus and Google Scholar

- Research which is freely available for redistribution

Submit your manuscript at www.biomedcentral.com/submit
C Biomed Central 